\title{
AN INDECOMPOSABLE DANIELL INTEGRAL
}

\author{
B. R. F. JEFFERIES AND D. H. FREMLIN
}

(Communicated by J. Jerry Uhl, Jr.)

Abstract. An example of an indecomposable Daniell integral is given.

0. Introduction. There is a useful approach to the integration of real-valued functions on a set $X$, alternate to integrating with respect to a measure, defined on a $\sigma$-algebra of subsets of $X$. It goes as follows.

Suppose that $L(X)$ is a vector lattice of real-valued functions $f: X \rightarrow \mathbf{R}$; that is, $L(X)$ is a vector space under pointwise addition and scalar multiplication, and the function $|f|: x \mapsto|f(x)|, x \in X$, belongs to $L(X)$ whenever $f$ does.

A linear mapping $u: L(X) \rightarrow \mathbf{R}$ is called a Daniell integral if $u(|f|) \geq 0$ whenever $f \in L(X)$, and if $f_{n} \in L(X), n=1,2, \ldots$, is a sequence of functions decreasing pointwise to zero, then $\lim _{n \rightarrow \infty} u\left(f_{n}\right)=0$.

It may be assumed from the outset that a given Daniell integral $u: L(X) \rightarrow \mathbf{R}$ is defined on a vector lattice $L(X)$ which is complete with respect to the seminorm $f \mapsto u(|f|), f \in L(X)$; if not, then the vector lattice can be completed in the standard way. Then there exists a (not necessarily finite) measure space $(X, \Sigma, \mu)$ such that $L(X)$ coincides with the family $\mathcal{L}^{1}(X, \Sigma, \mu)$ of all $\mu$-integrable functions and

$$
u(f)=\int_{X} f d \mu \text { for all } f \in L(X)
$$

provided that the vector lattice $L(X)$ satisfies Stone's condition (that is, it is a Stone vector lattice): the function $x \mapsto \min (f(x), 1), x \in X$, belongs to $L(X)$ whenever $f$ does.

The unique measure space $(X, \Sigma, \mu)$ for which (1) holds and $\Sigma$ is the $\sigma$-algebra generated by $L(X)$ is then easily characterized [2, 71G].

However, if $L(X)$ is not a Stone vector lattice, then it contains too few functions to determine a representing measure $\mu$ for which (1) holds. Furthermore, there are Daniell integrals, even with stronger convergence properties, which do not have any representing measures at all [4].

Nevertheless, there are still interesting examples of Daniell integrals on vector lattices failing Stone's condition which $d o$ have representing measures. For example, Kluvánek [7] has shown that a conical measure defined on a vector lattice of functions on a product of real lines has a decomposable representing measure. Incidentally, Kluvánek's result has recently been shown to be relevant to the spectral theory of operators [8].

A gap in Kluvánek's proof was filled by R. Becker [1], who also showed that for any Daniell integral $u: L(X) \rightarrow \mathbf{R}$ (completed as before), there exists a family

Received by the editors December 27, 1984.

1980 Mathematics Subject Classification (1985 Revision). Primary 28C05. 
of functions $f_{\iota} \in L(X), \iota \in I$, sets $X_{\iota} \subset X, \iota \in I$, and finite measure spaces $\left(X_{\iota}, \Sigma_{\iota}, \mu_{\iota}\right), \iota \in I$, such that $f_{\iota}(x)>0$ for all $x \in X_{\iota}, f / f_{\iota}$ is $\mu_{\iota}$-integrable on $X_{\iota}$ for every $f \in L(X)$, and

$$
u(f)=\sum_{\iota \in I} \int_{X_{\iota}} f / f_{\iota} d \mu_{\iota} \quad \text { for all } f \in L(X) .
$$

Thus by enlarging the set $X$ to the disjoint sum $\bigcup_{\iota \in I} X \times\{\iota\}$, the Daniell integral $u$ can be represented by a direct sum of measures with local changes of scale.

In Kluvánek's example [7], there is no need to enlarge the set $X$, the sets $X_{\iota} \subset X$, $\iota \in I$, are already pairwise disjoint, and for each $\iota \in I$, the function $f_{\iota}$ is identically one on $X_{\iota}$. It is, therefore, natural to ask if the representation (2) is always possible for a pairwise disjoint family of sets $X_{\iota}, \iota \in I$, finite measure spaces $\left(X_{\iota}, \Sigma_{\iota}, \mu_{\iota}\right)$, $\iota \in I$ and functions $f_{\iota}$ positive on $X_{\iota}$ for each $\iota \in I$. If such a representation is possible for a Daniell integral $u$, then $u$ is said to be decomposable. If not, then it is said to be indecomposable. The problem can be viewed as finding a family of pairwise disjoint local units for the vector lattice $L(X)$ sufficient to determine $u$.

An example of an indecomposable Daniell integral on a Stone vector lattice is given in $\S 1$.

1. An indecomposable Daniell integral. The task of constructing an indecomposable Daniell integral is related to the problem considered in [3] of enlarging a measure space to make it decomposable.

A complete, locally determined, Maharam measure space $(X, \Sigma, \mu)$ (see [3] for the terminology) for which there exists no decomposable measure space $(X, \Gamma, \nu)$ with $\Sigma \subset \Gamma$ and $\nu \mid \Sigma=\mu$ is produced in [3, Example 11]. However the example does depend on axioms known to be consistent with the usual axioms of set theory, and moreover, it is eliminated under the generalized continuum hypothesis. Similar techniques are useful in the present context, but there is no need to resort to special axioms.

Let $\omega_{1}$ be the first uncountable ordinal. A set $F \subset \omega_{1}$ is closed (in the order topology of $\omega_{1}$ ) if and only if $\sup B \in F$ for all nonempty countable subsets $B$ of $F$. A subset of $\omega_{1}$ is said to be bounded if it is contained in some order interval $[0, \beta], \beta<\omega_{1}$. Clearly, a subset of $\omega_{1}$ is bounded if and only if it is countable.

A closed and unbounded (that is, uncountable) subset of $\omega_{1}$ is termed a $c l u b$. A set $A \subset \omega_{1}$ is said to be stationary if $A \cap F \neq \varnothing$ for all clubs $F \subset \omega_{1}$. Therefore, $A \subset \omega_{1}$ is nonstationary whenever there exists a club $F \subset \omega_{1}$ such that $A \cap F=\varnothing$.

EXAMPLE. There exists a set $X$, a Stone vector lattice $L(X)$ of functions on $X$, and a Daniell integral $u: L(X) \rightarrow \mathbf{R}$ (with $L(X)$ completed as before) such that $u$ is indecomposable.

PROOF. Let $X=\omega_{1}$. Let $\omega_{2}$ be the least ordinal of greater cardinality than $\aleph_{1}$. First we show that there exists a family $\left\langle A_{\alpha}\right\rangle_{\alpha<\omega_{2}}$ of uncountable nonstationary subsets of $X$ such that $A_{\alpha} \cap A_{\beta}$ is countable whenever $\alpha<\beta<\omega_{2}$.

The sets $A_{\alpha}, \alpha<\omega_{2}$, are constructed recursively. Start with any uncountable, nonstationary subset of $\omega_{1}$; they do exist. Suppose that $\alpha<\omega_{2}$ and the collection $\left\langle A_{\beta}\right\rangle_{\beta<\alpha}$ of uncountable nonstationary subsets with the required property is given. We need to construct $A_{\alpha}$. 
If $\alpha=0$, set $F=\omega_{1}$; otherwise express $\alpha$ as $\left\{\beta_{\xi}: \xi<\omega_{1}\right\}$. For each $\xi<\omega_{1}$, the set $A_{\beta_{\xi}}$ is nonstationary, so there exists a club $F_{\xi}$ such that $A_{\beta_{\xi}} \cap F_{\xi}=\varnothing$. The set

$$
F=\left\{\xi: \xi<\omega_{1}, \xi \in F_{\eta} \quad \text { for all } \eta<\xi\right\}
$$

is a club. The proof is standard [5, Lemma 7.5], but it is reproduced here to make the argument as self-contained as possible.

To see that $F$ is closed, let $B$ be a nonempty countable subset of $F$ and set $\varsigma=\sup B$. Thus $\varsigma<\omega_{1}$. Let $\eta<\varsigma$. Suppose that $\varsigma \notin F_{\eta}$. Then because $F_{\eta}$ is closed, there exists $\theta<\varsigma$ such that $F_{\eta}$ does not meet $[\theta, \varsigma]$. Let $\xi$ be an element of $B$ such that $\max (\eta, \theta)<\xi \leq \varsigma$. But $B \subset F$, so $\xi \in F, \eta<\xi$ and $\xi \notin F_{\eta}$, which is a contradiction. Therefore, $\varsigma \in F_{\eta}$ for all $\eta<\varsigma$, so $\varsigma \in F$.

The set $F$ is seen to be unbounded in the following way. Let $\xi_{0}<\omega_{1}$. Define $\xi_{n}, n=1,2, \ldots$, recursively by

$$
\xi_{n+1}=\min \left(\bigcap_{\eta \leq \xi_{n}} F_{\eta} \backslash\left[0, \xi_{n}\right]\right), \quad n=0,1, \ldots
$$

It is easily checked that $\xi_{n} \leq \xi_{n+1}<\omega_{1}$ for all $n=0,1, \ldots$ Set $\xi=\sup \left\{\xi_{n}: n=\right.$ $0,1, \ldots\}$. If $\eta<\xi$, then there exists a number $n=0,1, \ldots$ such that $\eta<\xi_{n}$, and by the definition of $\xi_{m}$, we have $\xi_{m+1} \in F_{\eta}$ for all $m \geq n$. Since $F_{\eta}$ is closed, $\xi \in F_{\eta}$. As $\eta<\xi$ is arbitrary, $\xi \in F$. Furthermore, $\xi_{0}<\omega_{1}$ is arbitrary and $\xi_{0}<\xi$, so $F$ is unbounded.

The intersection of the set $F$ with each set $A_{\beta_{\xi}}, \xi<\omega_{1}$, is countable. To see this, let $\xi<\omega_{1}$. Then $A_{\beta_{\xi}} \cap F_{\xi}=\varnothing$, so $F \cap A_{\beta_{\xi}} \subset F \backslash F_{\xi}$. If $\varsigma \in F \backslash F_{\xi}$, then $\varsigma \in F_{\eta}$ for all $\eta<\varsigma$ and $\varsigma \notin F_{\xi}$, so we must have $\varsigma \leq \xi+1$. Therefore, $F \backslash F_{\xi}$ is countable and so is $F \cap A_{\beta_{\xi}}$.

The set $A_{\alpha}$ we are seeking can now be taken to be any uncountable nonstationary subset of $F$. Such sets exist; for example, let $\langle\theta(\xi)\rangle_{\xi<\omega_{1}}$ be the increasing enumeration of $F$, and put $A_{\alpha}=\left\{\theta(\xi+1): \xi<\omega_{1}\right\}$. Then because $F$ is closed, the set

$$
\left\{\theta(\xi): \xi<\omega_{1} \text { is a limit ordinal }\right\}
$$

is a club not intersecting $A_{\alpha}$, so $A_{\alpha}$ is nonstationary.

The required Daniell integral is the integral with respect to a measure $\mu$ defined as follows. Let $\Sigma$ be the $\sigma$-algebra of all subsets $E$ of $X$ such that either $E \cap A_{\alpha}$ is countable or $A_{\alpha} \backslash E$ is countable for every $\alpha<\omega_{2}$. For each $E \in \Sigma$, write

$$
P_{E}=\left\{\alpha<\omega_{2}: E \cap A_{\alpha} \text { is uncountable }\right\},
$$

and set $\mu(E)$ equal to the number of elements of the set $P_{E}$ whenever it is finite, and $\infty$ otherwise. We must check that $\mu$ is additive; that is, $P_{E} \cap P_{F}=\varnothing$ whenever $E, F \in \Sigma$ and $E \cap F=\varnothing$.

Suppose then, that $E, F \in \Sigma, E \cap F=\varnothing$, and $\alpha \in P_{E} \cap P_{F}$. Then $E \cap A_{\alpha}$ is uncountable and $F \cap A_{\alpha}$ is uncountable. Since $E \in \Sigma, A_{\alpha} \backslash E$ must be countable. But because $E \cap F=\varnothing$, we have $F \cap A_{\alpha} \subset A_{\alpha} \backslash E$, contradicting the uncountability of $F \cap A_{\alpha}$. Therefore, $P_{E} \cap P_{F}=\varnothing$ as required.

The additive set function $\mu: \Sigma \rightarrow[0, \infty]$ is clearly $\sigma$-additive. Let $L(X)$ be the space of all $\mu$-integrable functions and define the Daniell integral $u: L(X) \rightarrow \mathbf{R}$ by

$$
u(f)=\int_{X} f d \mu, \quad f \in L(X) .
$$


Now suppose that there exists a family of pairwise disjoint sets $X_{\iota}, \iota \in I$, functions $f_{\iota} \in L(X), \iota \in I$, and finite measure spaces $\left(X_{\iota}, \Sigma_{\iota}, \mu_{\iota}\right), \iota \in I$, such that for each $\iota \in I, f_{\iota}(x)>0$ for all $x \in X_{\iota}$ and $\left(f / f_{\iota}\right) \mid X_{\iota}$ is $\mu_{\iota}$-integrable, and the equality

$$
u(f)=\sum_{\iota \in I} \int_{X_{\iota}} f / f_{\iota} d_{\mu_{\iota}}
$$

holds for all $f \in L(X)$. The sum is absolutely convergent. It may be assumed that for each $\iota \in I,\left(X_{\iota}, \Sigma_{\iota}, \mu_{\iota}\right)$ is a complete measure space. For each $\iota \in I$, the smallest $\sigma$-algebra of subsets of $X_{\iota}$ for which each function $\left(f / f_{\iota}\right) \mid X_{\iota}, f \in L(X)$, is measurable is actually the smallest $\sigma$-algebra $\Gamma_{\iota}$ of subsets of $X_{\iota}$ for which each function $f \mid X_{\iota}, f \in L(X)$, is measurable; therefore $\Gamma_{\iota} \subset \Sigma_{\iota}$.

The characteristic function of a set $A \subset X$ is denoted by $\chi_{A}$. For each $\iota \in I$, $j=1,2, \ldots$, set

$$
X_{(\iota, j)}=\left\{x \in X: j-1<f_{\iota}(x) \leq j\right\} ; \quad \mu_{(\iota, j)}=\left(\chi_{X_{(\iota, j)}} / f_{\iota}\right) \mu_{\iota} .
$$

Then the sets $X_{(\iota, j)},(\iota, j) \in I \times \mathbf{N}$ are pairwise disjoint, and

$$
u(f)=\sum_{(\iota, j) \in I \times \mathbf{N}} \int_{X_{(\iota, j)}} f d \mu_{(\iota, j)}, \quad f \in L(X) .
$$

Now let $K=I \times \mathrm{N}$ and let the measure space $(X, \Gamma, \nu)$ be the direct sum of the finite measure spaces $\left(X_{\kappa}, \Sigma_{\kappa}, \mu_{\kappa}\right), \kappa \in K$; that is,

$$
\begin{gathered}
\Gamma=\left\{E \subset X: E \cap X_{\kappa} \in \Sigma_{\kappa} \text { for each } \kappa \in K\right\}, \\
\nu(E)=\sum_{\kappa \in K} \mu_{\kappa}\left(E \cap X_{\kappa}\right), \quad E \in \Gamma .
\end{gathered}
$$

For each $(\iota, j) \in K$, the $\sigma$-algebra $\Sigma_{(\iota, j)}$ is the collection of all sets $E \cap X_{(\iota, j)}$, $E \in \Sigma_{\iota}$. Then $\nu(E)=\mu(E)$ whenever $E \in \Sigma$ and $\mu(E)<\infty$. Because the measure spaces $(X, \Gamma, \nu)$ and $(X, \Sigma, \mu)$ are both semifinite, their magnitudes are the same [3]. Therefore, the cardinality of the set $K$ is $\aleph_{2}$. However, there cannot be $\aleph_{2}$ disjoint subsets of $\omega_{1}$, so this is a contradiction. The Daniell integral $u$ is therefore indecomposable.

The example is certainly pathological, but it is not the purpose of this note to analyze its pathology. The notion of a compact vector lattice of functions [6] may be useful for showing that a given Daniell integral is decomposable.

ACKNowledgment. One of us (B.R.F.J.) would like to thank R. Becker for pointing out an error in an earlier "proof" that every Daniell integral is decomposable.

The referee kindly suggested the following shorter construction of the collection $\left\langle A_{\alpha}\right\rangle_{\alpha<\omega_{2}}$.

There exists a disjoint collection of sets $D$ in $X$ such that

(1) $A \in D$ implies $|A|=|X|$, and

(2) $|D|=|X|$.

By Zorn's lemma there exists a maximal collection of sets $C$ in $X$ such that

(i) $D \subset C$,

(ii) $A, B \in C$ and $A \neq B$ implies $A \cap B$ is countable, and

(iii) $A \in C$ implies $|A|=|X|$. 
We shall see that $|C|=\aleph_{2}$. If not, then since $D \subset C,|C|=\aleph_{1}$. Thus the collection $C$ may be indexed by $\omega_{1}$, so that $C=\left\{A_{\alpha}: \alpha \in \omega_{1}\right\}$. Let $B_{\alpha}=A_{\alpha} \backslash \bigcup_{\beta<\alpha} A_{\alpha}$. Then $B_{\alpha} \subset A_{\alpha}$ and $B_{\alpha} \backslash A_{\alpha}$ is countable for all $\alpha \in \omega_{1}$. Also, the collection $\left\{B_{\alpha}: \alpha \in \omega_{1}\right\}$ is a disjoint collection of sets each with cardinality $\aleph_{1}$. Now choose a point from each set $B_{\alpha}$ and call the resulting set $A$. It is easy to see that $A_{\alpha} \cap A$ is countable for each $\alpha \in \omega_{1}$, and $|A|=\aleph_{1}$. This contradicts the maximality of $C$. Hence $|C|=\aleph_{2}$.

\section{REFERENCES}

1. R. Becker, Sur l'intégrale de Daniell, Rev. Roumaine Math. Pures Appl. 26 (1981), 189-206.

2. D. H. Fremlin, Topological Riesz spaces and measure theory, Cambridge Univ. Press, Cambridge, 1974.

3. __ Decomposable measure spaces, Z. Wahrsch. Verw. Gebiete 45 (1978), 159-167.

4. D. H. Fremlin and M. Talagrand, On the representation of increasing linear functionals on Riesz spaces by measures, Mathematika 25 (1978), 213-215.

5. T. Jech, Set theory, Academic Press, New York, 1978.

6. B. Jefferies, Semicompact functionals, Rev. Roumaine Math. Pures Appl. (in press).

7. I. Kluvánek, Conical measures and vector measures, Ann. Inst. Fourier (Grenoble) 27 (1977), 83-105.

8. W. Ricker, On Boolean algebras of projections and scalar-type spectral operators, Proc. Amer. Math. Soc. 87 (1983), 73-77.

Department of Mathematics, Ohio State University, 231 West 18th Avenue, Columbus, OHIO 43210

Department of Mathematics, University of Essex, Wivenhoe Park, ColCHESTER CO4 3SQ, ENGLAND (Current adress of D. H. Fremlin)

Current address (B. R. F. Jefferies): School of Mathematics and Physics, Macquarie University, North Ryde, N.S.W. 2113, Australia 